\title{
A Case of Reed's Syndrome: An Underdiagnosed Tumor Disorder
}

\author{
Georgios Kontochristopoulos ${ }^{\mathrm{a}}$ Anargyros Kouris $^{\mathrm{a}} \quad$ Evgenia Balamoti $^{\mathrm{a}}$ \\ Charitomeni Vavoulia $^{\mathrm{a}}$ Vasiliki Markantoni $^{\mathrm{a}} \quad$ Elefteria Christofidou $^{\mathrm{b}}$ \\ Christina Antoniou ${ }^{\mathrm{a}}$ \\ Departments of a Dermatology and Venereology and ${ }^{b}$ Dermatopathology, 'Andreas \\ Sygros' Hospital, Athens, Greece
}

\section{Key Words}

Reed's syndrome · Cutaneous leiomyoma · Uterine fibroid

\begin{abstract}
Cutaneous leiomyomas are uncommon, benign smooth muscle tumors originating from the arrector pili muscle of the hair follicle that are frequently unrecognized and underdiagnosed by clinicians. They sometimes coexist with common uterine fibroids in an inherited disorder named multiple cutaneous and uterine leiomyomatosis, also referred to as Reed's syndrome. We report a case of Reed's syndrome in a young woman who had been misdiagnosed for many years.

(c) 2014 S. Karger AG, Basel
\end{abstract}

\section{Introduction}

Reed's syndrome (RS) is an autosomal dominant genetic disorder characterized by cutaneous and uterine leiomyomas, initially described in 1954 in a 45-year-old woman [1]. In 1973, Reed et al. [2] reported on the members of two families whose successive generations demonstrated cutaneous leiomyomas, uterine leiomyomas and/or leiomyosarcomas, establishing an autosomal dominant pattern of inheritance. Affected females frequently develop uterine leiomyomas that are larger and more numerous and emerge earlier than those seen in the general population. A subset of patients with RS is predisposed to developing papillary renal cell carcinoma, necessitating evaluation of all patients for the presence of renal malignancy. 
Kontochristopoulos et al:: A Case of Reed's Syndrome: An Underdiagnosed Tumor Disorder

\section{Case Presentation}

A 38-year-old woman presented to our department with a cluster of papules and nodules on her right buttock. The disease had first manifested at the age of 14, but the lesions had almost doubled in number over the last 10 years. Clinical examination of the skin revealed multiple superficial, hyperpigmented, smooth dermal papules and nodules of varying size on the right buttock (fig. 1). The lesions were asymptomatic, apart from occasional slight pain induced by cold and heat. At the age of 28 , she presented pelvic pain and menorrhagia, indicating uterine involvement. Given that the consistent duration of her menstruation was 2 weeks, she was placed on gonadotropin therapy. Subsequently, she underwent surgery for uterine fibroids; a myomectomy was performed rather than hysterectomy in order to preserve fertility. Her medical history also included an appendectomy at the age of 15 following histological findings of a carcinoid.

Complete blood count analysis, urea, creatinine, electrolytes, antinuclear antibodies, anti-DNA, C3 and C4 complement as well as the results of liver function tests and chest radiography were normal. A skin biopsy revealed a pilar leiomyoma (piloleiomyoma). Histopathological examination showed a proliferation of smooth muscle bundles intermingled with varying amounts of collagen fibers and a few lymphocyte infiltrates (fig. 2). Due to the patient's history of myomectomy, pelvic ultrasonography and abdominal magnetic resonance imaging were performed which revealed uterine fibroids (fig. 3). Our patient finally underwent a hysterectomy, and the subsequent biopsy confirmed the diagnosis of uterine leiomyoma. A diagnosis of multiple cutaneous and uterine leiomyomatosis, typical for RS, was established. The lesions had remained misdiagnosed for many years.

\section{Discussion}

The principal dermatologic manifestation of RS is the presence of cutaneous leiomyomas. Clinically, they occur as small, smooth-surfaced, skin-colored or pinkish-brown, solitary and/or multiple papules or nodules that range from 0.2 to $2.0 \mathrm{~cm}$ in diameter [3]. Cutaneous leiomyomas are divided into three categories: piloleiomyomas arising from the arrector pili muscle of the hair follicle, genital leiomyomas emerging from the tunica dartos of the scrotum and the mammary muscles of the nipples, and angioleiomyomas originating from vascular smooth muscle $[4,5]$. They can be asymptomatic or painful at the time of appearance. The extremities are the most frequently involved sites, particularly the extensor surfaces, followed by the trunk, face and neck [3]. More than one area of the body can be involved in the majority of these patients, and the lesions develop in adolescence or late adulthood $[3,4]$.

Piloleiomyoma is the most common type of cutaneous leiomyoma, usually occurring in early adult life. The majority of them are tender or painful, the pain often being described as burning, pinching or stabbing $[5,6]$. Pain or tenderness may be spontaneous or induced by cold, stress, touch, trauma or pressure [7]. Many authors support the idea that pain or tenderness may be secondary to pressure on nerve fibers within the tumor, whereas others suggest that they could be due solely to contraction of muscle fibers [3].

Histopathology demonstrates smooth muscle fiber bundles with varying degrees of intermingled collagen in the dermis $[5,7,8]$. The smooth muscle fibers are composed of eosinophilic cytoplasm with elongated blunt-ended nuclei with little or no waviness [6]. Severe inflammation, significant cytologic atypia, necrosis or encapsulation are not appar- 
Kontochristopoulos et al.: A Case of Reed's Syndrome: An Underdiagnosed Tumor Disorder

ent. If necessary, desmin, smooth muscle actin and muscle-specific actin may highlight the smooth muscle origin of the tumor [4].

The predisposition to RS has been localized to the chromosome 1q42.3-q43 $[9,10]$ in the gene encoding fumarate hydratase (FH). FH converts fumarate to malate in the Krebs cycle, and is also thought to act as a suppressor of tumor genes [10-12]. Association of this syndrome with renal cell carcinoma has recently been highlighted $[13,14]$. The syndrome of hereditary leiomyomatosis and renal cell carcinoma also results from a germline mutation in the gene encoding FH $[11,12]$. The incidence of renal cell carcinoma in people with FH mutation, though unclear, seems to be lower than that documented for uterine and cutaneous leiomyomatosis. Some reports refer to an incidence of $<1-2 \%$. Renal cell carcinoma associated with RS occurs in the third to fourth decade of life and histopathologically demonstrates cysts of papillary cell carcinoma [15]. This carcinoma generally follows an aggressive course with early metastasis. Our patient did not develop renal cell carcinoma. Nonetheless, she underwent an appendectomy due to the carcinoid. The possibility of an association between carcinoids, which are benign tumors, and RS has not yet been considered in the literature, but warrants investigation.

Toro et al. [13] reported that patients with RS developed cutaneous lesions at a statistically significantly ( $p=0.048$ ) earlier mean age (25 years) in comparison with the mean age at diagnosis of uterine leiomyomas (30 years). In our case, the onset of cutaneous leiomyomas began after puberty, at the age of 14 . According to a study of a large case series conducted by Alam et al. [6], the mean age of onset is 24.1 years (median 25 years, range 945 years), while in a minority of female patients, the onset correlated with hormonal change, such as puberty, pregnancy, a contraceptive regimen or other hormonal medication. Kim [8] reported a case of skin lesions appearing in a 51-year-old woman after the initiation of estrogen replacement therapy following an oophorectomy for ovarian cysts. In our case, the patient was diagnosed with fibroids at 28 years of age; she experienced several gynecological symptoms and underwent a myomectomy. She had been misdiagnosed for several years before being admitted to our department at 38 years of age where she was diagnosed as having RS.

The treatment of cutaneous leiomyomas depends on the number of lesions and the presence or absence of symptoms. Camouflage with cosmetics and avoidance of cold may be sufficient for some patients [7]. Medications such as oral nitroglycerin, nifedipine, doxazosin and phenoxybenzamine, which block smooth muscle contraction, and medications that target nerve activity, such as gabapentin and topical analgesics that have been used to treat symptomatic leiomyomas, have shown limited effectiveness [2, 5, 7, 8]. When few lesions are present, surgical excision with skin grafting can be considered [3]. Recurrences have been reported to occur from 6 weeks to more than 15 years after excision. Cryotherapy and electrocoagulation have also been shown to provide some benefit [3]. Notably, a recent report by Christenson et al. [16] described carbon dioxide laser ablation as an effective treatment for multiple cutaneous leiomyomas complete disappearance of pain symptoms 9 months after its application.

Although RS is quite rare, dermatologists should bear it in mind in patients with multiple leiomyomas because of the risk of severe uterine fibroids and in some cases aggressive renal cancer [17-22]. Even though cutaneous leiomyomas are benign tumors, women affected by this condition can develop uterine fibroids with pain, menorrhagia and infertility. Consequently, appropriate screening measures for associated disorders are mandatory. 
Kontochristopoulos et al:: A Case of Reed's Syndrome: An Underdiagnosed Tumor Disorder

\section{References}

1 Blum P, Jean L: Besnier's eruptive leiomyoma (in French). Bull Soc Fr Dermatol Syphiligr 1954;61:349-350.

-2 Reed WB, Walker R, Horowitz R: Cutaneous leiomyomata with uterine leiomyomata. Acta Derm Venereol 1973;53:409-416.

-3 Garman ME, Blumberg MA, Ernst R, et al: Familial leiomyomatosis: a review and discussion of pathogenesis. Dermatology 2003;207:210-213.

4 Spencer JM, Amonette RA: Tumors with muscle differentiation. Derm Surg 1996;22:761-768.

5 Holst VA, Junkins-Hopkins JM, Elenitsas R: Cutaneous smooth muscle neoplasms: clinical features, histologic findings, and treatment options. J Am Acad Dermatol 2002;46:477-490.

6 Alam NA, Barclay E, Rowan AJ, et al: Clinical features of multiple cutaneous and uterine leiomyomatosis. Arch Dermatol 2005;141:199-206.

7 Muret PG, Pujol RM, Alomar A, et al: Familial leiomyomatosis cutis et uteri (Reed's syndrome). Arch Dermatol Res 1988;280(suppl):S29-S32.

8 Kim G: Multiple cutaneous and uterine leiomyomatosis (Reed's syndrome). Dermatol Online J 2005;11:21.

-9 Martinez A, Gordon D, Horev L, et al: Multiple cutaneous and uterine leiomyomas: refinement of the genetic locus for multiple cutaneous and uterine leiomyomas on chromosome 1q42.3-43. J Invest Dermatol 2002;118:876-880.

10 Choudhary S, McLeod M, Torchia D, et al: Multiple cutaneous and uterine leiomyomatosis syndrome: a review. J Clin Aesthet Dermatol 2013;6:16-21.

11 Rongioletti F, Fausti V, Ferrando B, et al: A novel missense mutation in fumarate hydratase in an Italian patient with a diffuse variant of cutaneous leiomyomatosis (Reed's syndrome). Dermatology 2010;221: 378-380.

$\checkmark 12$ Parmentier L, Tomlinson I, Happle R, et al: Evidence for a new fumarate hydratase gene mutation in a unilateral type 2 segmental leiomyomatosis. Dermatology 2010;221:149-153.

-13 Toro JR, Nickerson ML, Wei MH, et al: Mutations in the fumarate hydratase gene cause hereditary leiomyomatosis and renal cell cancer in families in North America. Am J Hum Genet 2003;73:95-106.

14 Rothman A, Glen G, Choyke L, et al: Multiple painful cutaneous nodules and renal mass. J Am Acad Dermatol 2006;55:683-686.

15 Linehan WM, Walther MM, Zbar B: The genetic basis of cancer of the kidney. J Urol 2003;170:2163-2172.

16 Christenson LJ, Smith K, Aprey CJ: Treatment of multiple cutaneous leiomyomas with $\mathrm{CO}_{2}$ laser ablation. Dermatol Surg 2000;26:319-322.

17 Mandal RK, Koley S, Banerjee S, et al: Familial leiomyomatosis cutis affecting nine family members in two successive generations including four cases of Reed's syndrome. Indian J Dermatol Venereol Leprol 2013;79:83-87.

18 Michelon MA, Layton CJ, Jessup CJ, et al: Cutaneous clues to renal cell carcinoma: hereditary leiomyomatosis and renal cell carcinoma. J Drugs Dermatol 2013;12:578-579.

19 Henley ND, Tokarz VA: Multiple cutaneous and uterine leiomyomatosis in a 36-year-old female, and discussion of hereditary leiomyomatosis and renal cell carcinoma. Int J Dermatol 2012;51:1213-1216.

20 Mitchum MD, Adams EG, Holcomb KZ: JAAD Grand Rounds quiz. A 46-year-old man with agminated papules on the buttock. Reed syndrome. J Am Acad Dermatol 2012;66:337-339.

21 Elbuluk N, Bichakjian CK, Lowe L: What is your diagnosis? Multiple cutaneous and uterine leiomyomatosis (Reed syndrome). Cutis 2011;87:65, 76-77.

22 Emer JJ, Solomon S, Mercer SE: Reed's syndrome: a case of multiple cutaneous and uterine leiomyomas. J Clin Aesthet Dermatol 2011;4:37-42. 
Kontochristopoulos et al:: A Case of Reed's Syndrome: An Underdiagnosed Tumor Disorder

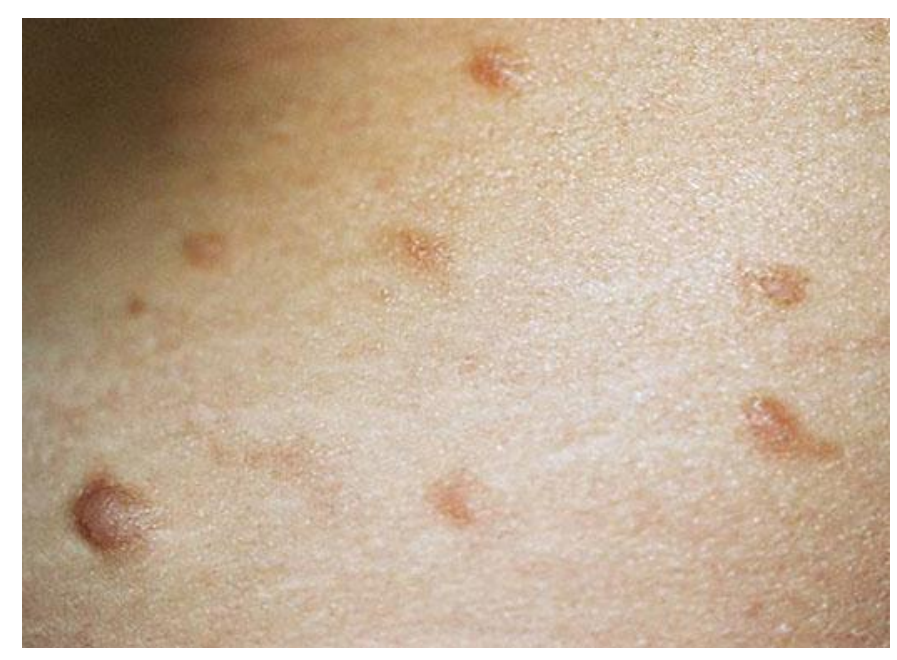

Fig. 1. Collection of dusky pink-brown firm papules and nodules of varying sizes.
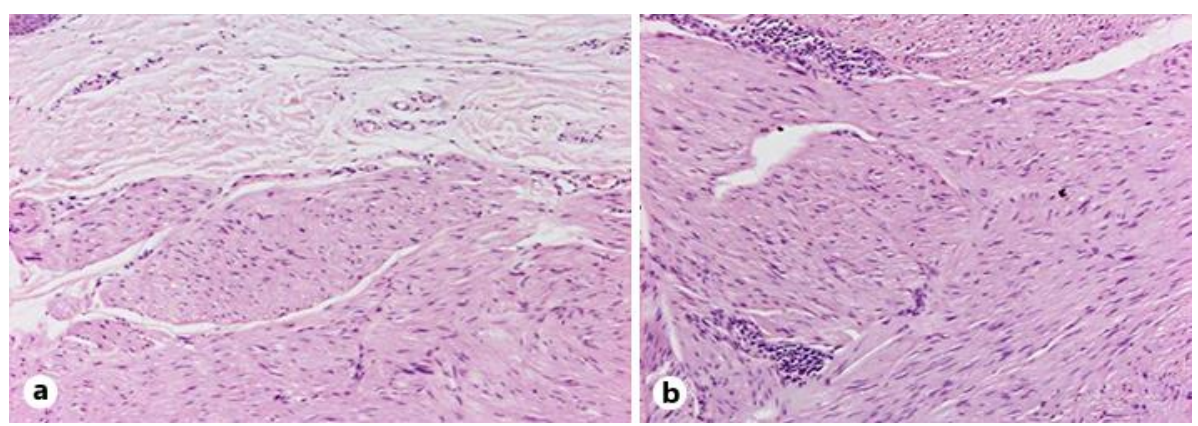

Fig. 2. a Microscopic histological view of a skin biopsy specimen. The neoplasm consisted of poorly demarcated interlacing bundles of smooth muscle fibers (H\&E, $\times 40)$. b The above smooth muscle fibers intermingled with varying amounts of collagen bundles and a few lymphocyte infiltrates $(H \& E, \times 100)$.
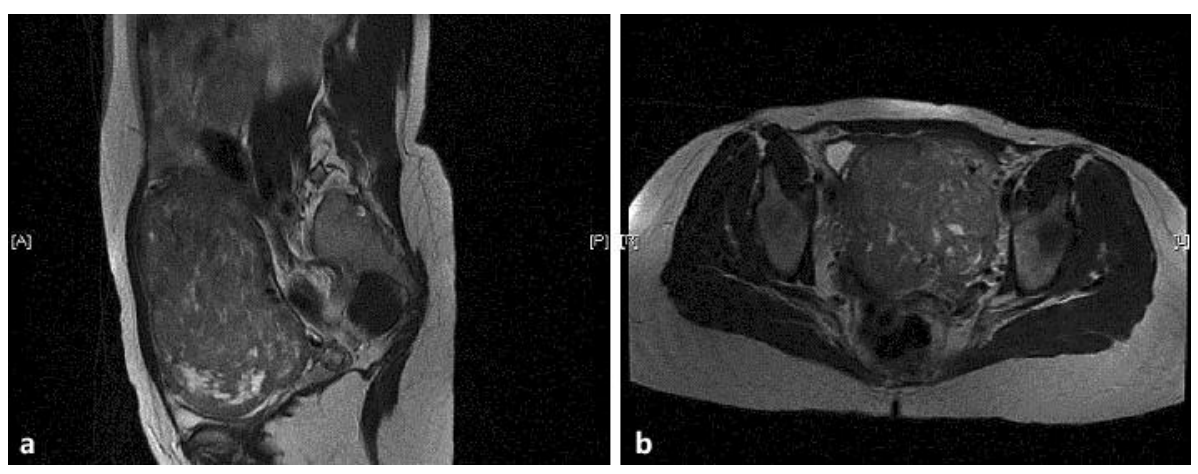

Fig. 3. Abdominal magnetic resonance image at time of diagnosis showed uterine fibroids. a Axial T2weighted lower abdomen. b Sagittal T2-weighted lower abdomen. 